\title{
INTEGRATION AND IN-FIELD GAINS SELECTION OF FLIGHT AND NAVIGATION CONTROLLER FOR REMOTELY PILOTED AIRCRAFT SYSTEM
}

\author{
Maciej SŁOWIK*, Daniel OŁDZIEJ*; Zdzisław GOSIEWSK* \\ "Moose sp z. 0.0., Żurawia 71 Street, 15-540 Bialystok, Poland \\ *Bialystok University of Technology, Faculty of Mechanical Engineering, Wiejska 45C Street, 15-351 Bialystok, Poland \\ maciej.slowik@mooseinc.eu, d.oldziej@pb.edu.pl, z.gosiewski@pb.edu.pl
}

received 10 October 2015, revised 23 February 2017, accepted 6 March 2017

\begin{abstract}
In the paper the implementation process of commercial flight and navigational controller in own aircraft is shown. The process of autopilot integration were performed for the fixed-wing type of unmanned aerial vehicle designed in high-wing and pull configuration of the drive. The above equipment were integrated and proper software control algorithms were chosen. The correctness of chosen hardware and software solution were verified in ground tests and experimental flights. The PID controllers for longitude and latitude controller channels were selected. The proper deflections of control surfaces and stabilization of roll, pitch and yaw angles were tested. In the next stage operation of telecommunication link and flight stabilization were verified. In the last part of investigations the preliminary control gains and configuration parameters for roll angle control loop were chosen. This enable better behavior of UAV during turns. Also it affected other modes of flight such as loiter (circle around designated point) and auto mode where the plane executed a pre-programmed mission.
\end{abstract}

Key words: Remotely Piloted Aircraft System, Unmanned Aerial Vehicle, Autopilot, Flight Controller, Navigation Controller

\section{INTRODUCTION}

In the last five years rapid development of different technologies such as formation flight (Kownacki and Ołdziej, 2016; Gosiewskia and Ambroziak, 2013) or launchers for take-off (Kondratiuk and Gosiewski, 2013), connected with unmanned aerial vehicles (UAVs) can be observed. They become standard in different military and civilian applications. Among them, assessment of crisis situations, monitoring of mass ventures, recording videos and taking photos from the air can be distinguished (Marconi et al., 2011; Erdos et al., 2013). But still there is a constant need for the human operator who control air vehicle. It force the investigations connected with replacing of the operator by automatic control system (Koszewnik, 2014; Spinka et al., 2011). Used autopilots consist of sensor modules which provide stabilization of flight (such as gyroscopes, accelerometers and magnetometers) and facilitate the navigation (such as global navigational satellite signals (GNSS) receivers and compasses). These elements are the basis for calculating the orientation and position in space (Walendziuk et al., 2015). Among available solutions two groups of autopilots can be distinguished: the first one have closed firmware, the second one - have open-source code which is almost always supported by hobbyist and science community (Mahony et al., 2012; Arifianto and Farhood, 2015; Meier et al., 2012; HaiYang, 2010).

A methodology of the integration of market available component of UAV to prepare it for the autonomous flight is considered in the paper (Fig. 1). The properties of used aircraft equipment are described. After that the simulations of flight modes and control algorithms were tested by software in the loop (SITL). Next ground control station (GCS) adjusted to the aircraft was described. It allow for C2 (Communication and Control) mode of operation what allows to view, analyse and setting parameters of flight. It also enable control the mission of UAV, changing modes of flight, changes of waypoints and their altitude (Wang et al., 2006). Above mentioned simulations were used for correctness testing of algorithms and flight modes without risk of damaging of a real aircraft model. In the next stage of investigations the flight field tests were conducted, whereas trim and communication link tests were made. The experimental choice of controller gains for roll angle were performed. Next the impact of different gains on the aircraft during flight were shown.

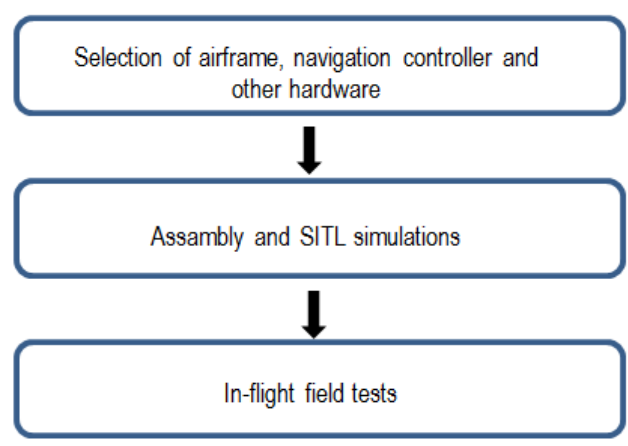

Fig. 1. Methodology scheme of the UAV integration

\section{HARDWARE}

As the vehicle for the whole system, aircraft in high wing configuration was used. The chosen airplane (Multiplex Mentor) is a micro class airframe - which MTOM (Maximum Take-Off Mass) is below 2 [kg] (Fig. 2) (Multiplex Mentor Assambly Manual - Tab. 1). 


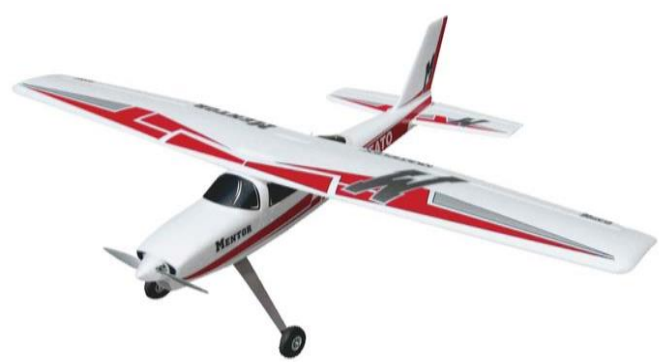

Fig. 2. Multiplex Mentor

The expanded polypropylene foam known as Elapor is structure material. Three-phase electrical engine is a propulsion drive. This type of drive for UAV allows for the distribution of power to all components of onboard systems such as autopilot, sensors, communication modules and drives of control surfaces.

Tab. 1. UAV fuselage specification (Multiplex Mentor Assambly Manual)

\begin{tabular}{|c|c|}
\hline Fuselage & Elapor foam \\
\hline Airfoil & High-wing placement \\
\hline Wingspan & $1650[\mathrm{~mm}]$ \\
\hline Wing area & $45\left[\mathrm{dm}^{2}\right]$ \\
\hline Wing loading & $44,5\left[\mathrm{~g} / \mathrm{dm}^{2}\right]$ \\
\hline Length & $1170[\mathrm{~mm}]$ \\
\hline Fuel type & electric \\
\hline $\begin{array}{c}\text { Weight (with 3s LiPo 3,2Ah } \\
\text { recommended battery) }\end{array}$ & $1950[\mathrm{~g}]$ \\
\hline
\end{tabular}

The drive and power elements shown in Tab. 2 are also constant elements of UAV.

Tab. 2. UAV components

\begin{tabular}{|l|l|l|}
\hline \multirow{4}{*}{ Main drive } & BLDC motor & $\begin{array}{l}\text { DUALSKY } \\
\text { XM3548CA-4 }\end{array}$ \\
\cline { 2 - 3 } & Propeller & APC 11x5,5 \\
\cline { 2 - 3 } & $\begin{array}{l}\text { Motor speed } \\
\text { controller }\end{array}$ & Emax ESC 50A \\
\hline \multirow{2}{*}{$\begin{array}{l}\text { Control surfaces } \\
\text { drives }\end{array}$} & Servo motors & Hitec HS 311 \\
\cline { 2 - 3 } & Servo motors & Hitec HS 82MG \\
\hline Power source & Rechargeable battery & LiPo 3S 3,6Ah \\
\hline Communication link & Pair of radiomodems & 3DR 433 [MHz] \\
\hline
\end{tabular}

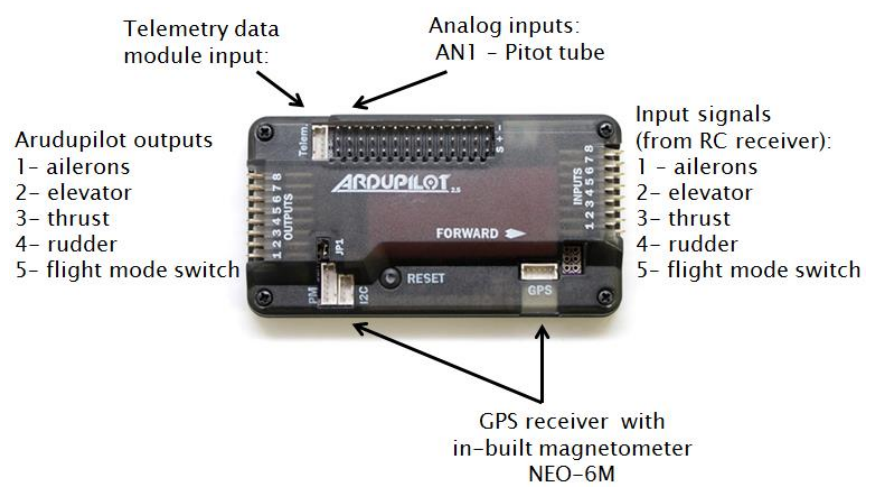

Fig. 3. The scheme of the autopilot inputs/outputs
In order to build fully efficient and functional unnamed system there is a need for use autopilot unit which can stabilize flight and control navigation. Ardupilot 2.6 shown in Fig. 3 (http://plane. ardupilot.com/) is a chosen autopilot.

Full functionality of autopilot can be achieved by using of the dedicated peripheral elements such as GPS receiver with magnetometer, static and dynamic pressure sensor, power module with current sensor and radio modem for communication with ground control station. Implementation of autopilot with above modules is shown in Fig. 4.

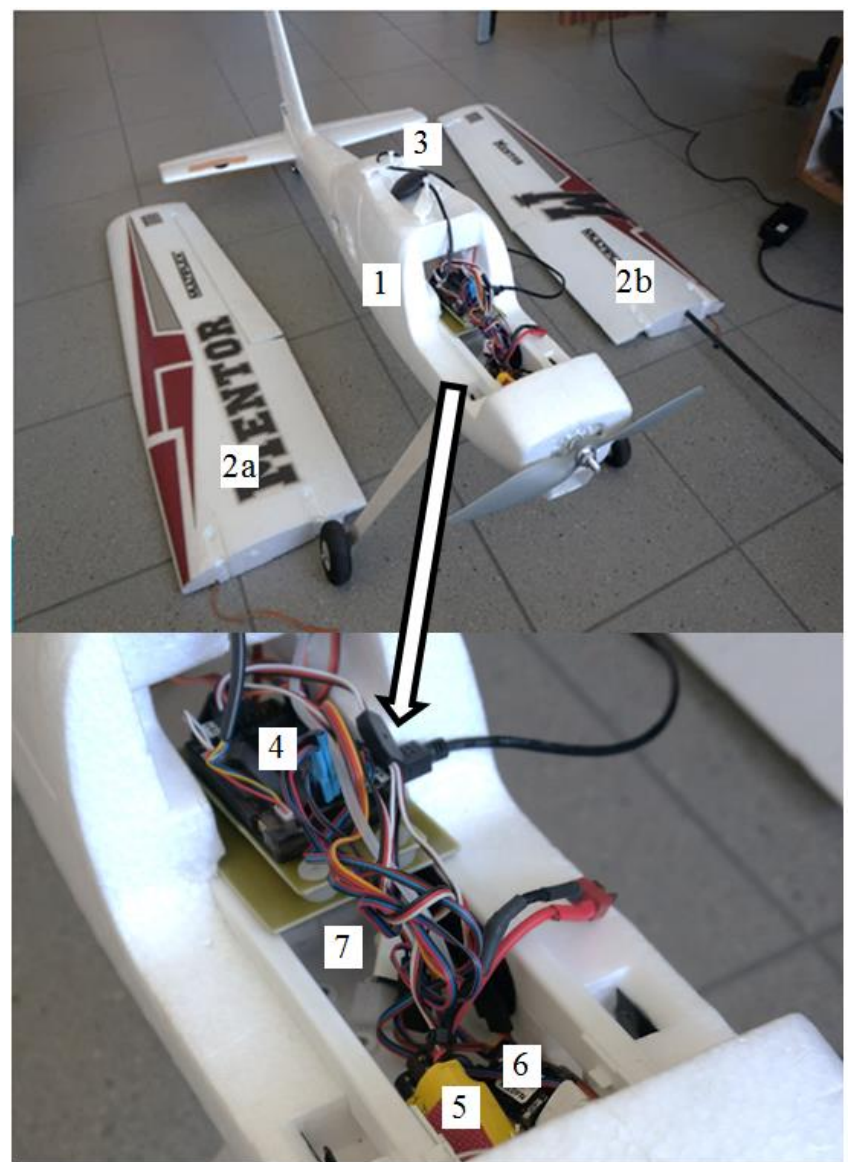

Fig. 4. Hardware assamblied on board of Mentor airframe. 1 - fuselage; $2 a$ - right part of wing, $2 b$ - left part of wing 3 - GPS receive, 4 - flight and navigation controller, 5 - motor speed controller, $6-\mathrm{RC}$ receiver, 7 - battery chamber

\section{SITL SIMULATIONS}

After configuration completion, the proper work of software and flight modes in software in the loop simulation have been tested. For this purpose the software package for emulation of autopilot, communication system (Mavlink protocol (Crespo et al., 2014)) and application for ground control station were chosen (http://planner.ardupilot.com/). For assembly of above mentioned the Cygwin package which enable utilities and compilers from GNU/Linux environment was installed. Next, compilation process was configured for firmware dedicated for planes - ArduPlane. Afterwards Mission Planner GCS was set for sending and receiving telemetry data frames (under TCP/UDP protocol) from and to virtual ArduPilot controller. Final version of software in the loop simulation consist of the following applications: mavproxy, console, map and mission planner. 


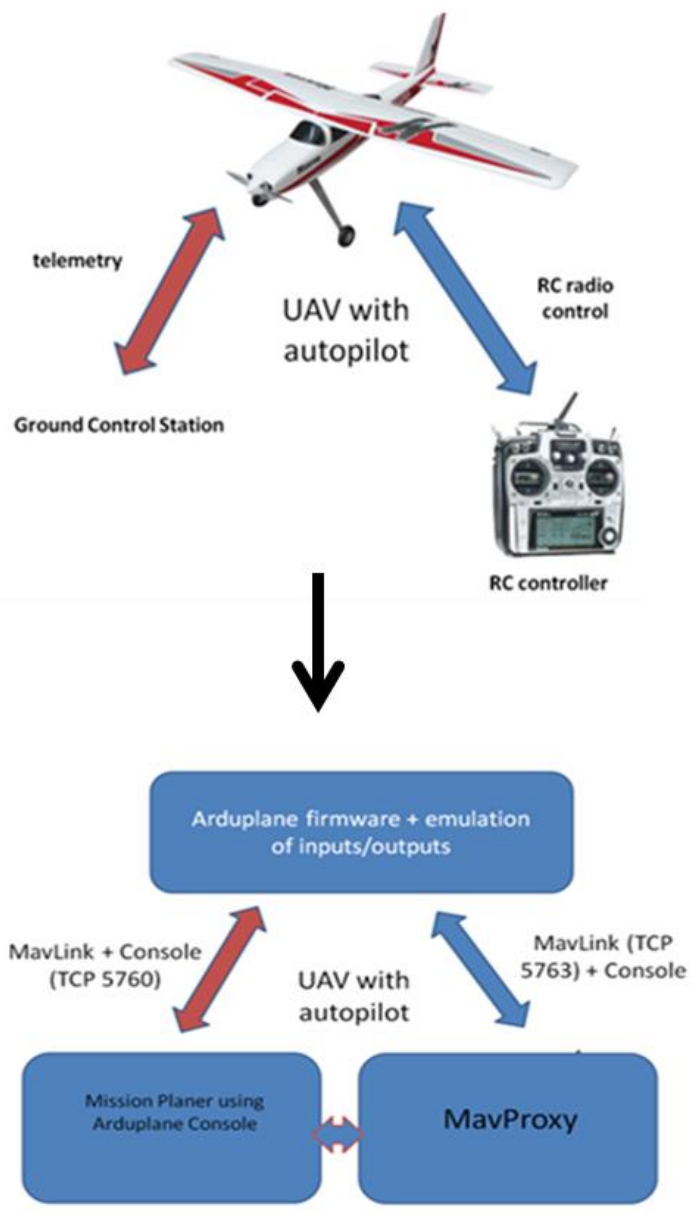

Fig. 5. Schematic diagram showing classic approach for control UAV with use of ArduPilot (up) and transition to SITL simulation (down) where functions of human operator with RC controller and GCS are executed by assembly of simulation programs with dedicated interfaces

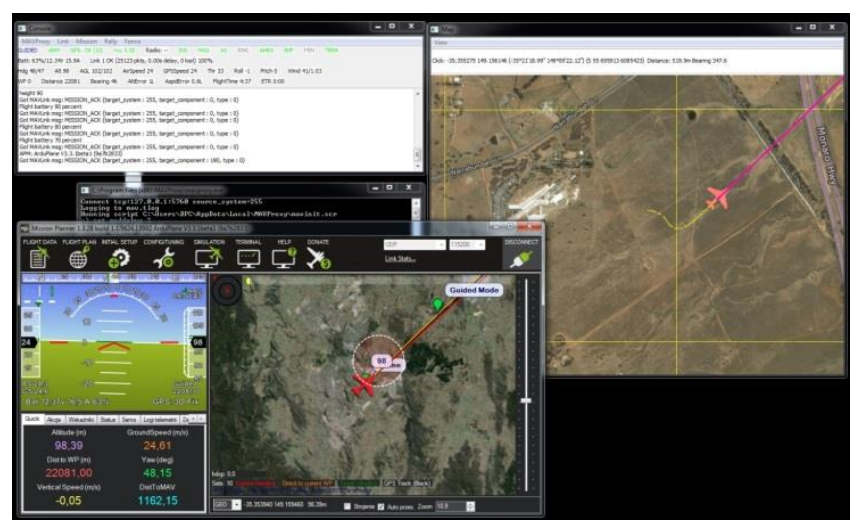

Fig. 6. The print screen showing SITL simulation. In the upper right corner - MavProxy, on the right side in the background the console application, in the lower right corner - GCS Mission Planner, on the left side - the map application - which allow view of current position of UAV on the map

During the investigations the different flight modes such as manual, stabilize, fly by wire, loiter and auto were checked. The schematic diagram of transforming from classical UAV control to SITL simulation was shown below in Fig. 5. The control link of RC controller and communication and control link from GCS were substituted by software solution. It enable use of MavProxy applications, which are proxy between elements of simulation system. Additionally in described simulation one can view and change low-level parameters of control surfaces deflection and engine throttle in the same way as in RC controller. With this solution, there exist a possibility of modification of flight parameters such as roll, pitch and yaw angle, geographical latitude and longitude or altitude. The simulation allows for setting waypoints for automatic flight and tuning gains of PID controllers responsible for flight stabilization and navigation (Fig. 6). Realization of above simulations faciliate the inflight field tests.

Currently the PID control is not the best control method, but is relevantly simple which impact on its implementation ability. It also allow for finding out object dynamic, which further open posibiity to use more complex and advanced controllers of flight parameters (Koslosky, 2015; Kownacki, 2015; Mystkowski, 2014).

\section{IN-FLIGT FIELD TESTS}

During the field experiments series of flight tests were made. At the beginning the final trimming of Mentor UAV was done. Further, modes of assisted manual flight were tested to ensure proper setting of controller parameters. The stabilize mode in which autopilot ensure limited level of deflection of control surfaces set by operator and stabilization based on IMU information was then tested.

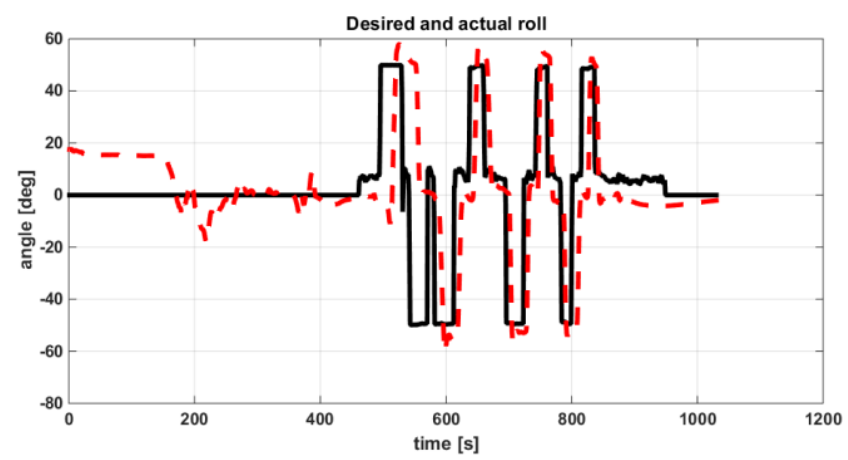

Fig. 7. Changes of roll angle during flight in FBWA mode with proportional gain of PID roll angle controller equals 0.4 . After 470 [s] the manual flight mode was changed for FBWA and rapid changes of bank angle (up to $50\left[^{\circ}\right]$ in both sides) was commanded - solid line desired value, dashed line - actual value

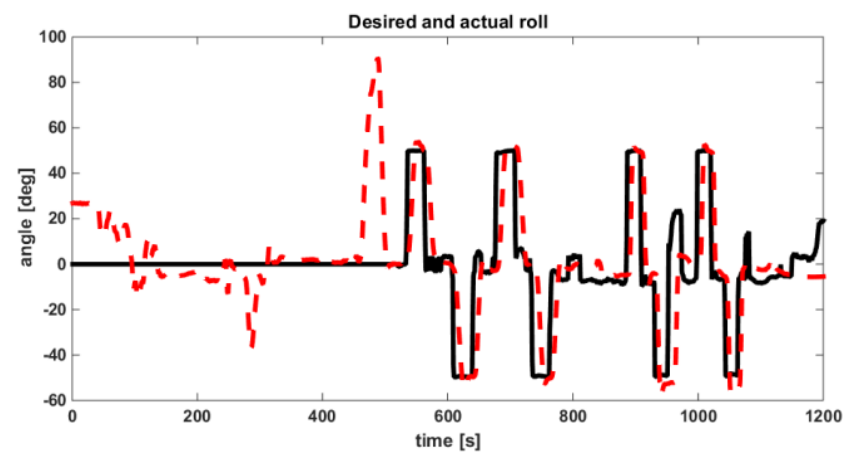

Fig. 8. Changes of roll angle during flight in fly by wire A mode with proportional gain of PID roll angle controller equals 1.4. After 530 [s] the manual flight mode was changed for FBWA and rapid changes of bank angle (up to $50\left[^{\circ}\right]$ in both sides) was commanded - solid line desired value, dashed line - actual value. - solid line desired value, dashed line - actual value 
The next FBWA (fly by wire type A) mode, where flight controller hold roll angle to maximal value of LIM_CD_ROLL parameter was tested. This enable safe manual turning in the air without risk of loss of altitude during this maneuver. The stall prevention parameter, which limited bank angle to $25\left[^{\circ}\right]$ was disabled to ensure possibility of narrower turns. The preliminary in-flight tuning of PID controller for roll was made. The proportional gain was changed increasingly from 0.4 , which was default value in tests, but object was under-steer. The parameter was changes up to 1.9 value, then object was over-steer and highly overshooted which complicated fluenty turning after course changes, especcialy over 30 degrees. Finally the gain was decreased to value 1.4. It caused faster reaction of airplane for commanded bank angle (shown in Fig. 7 and Fig. 8) which enable narrower turns and better behavior in different modes of flight.

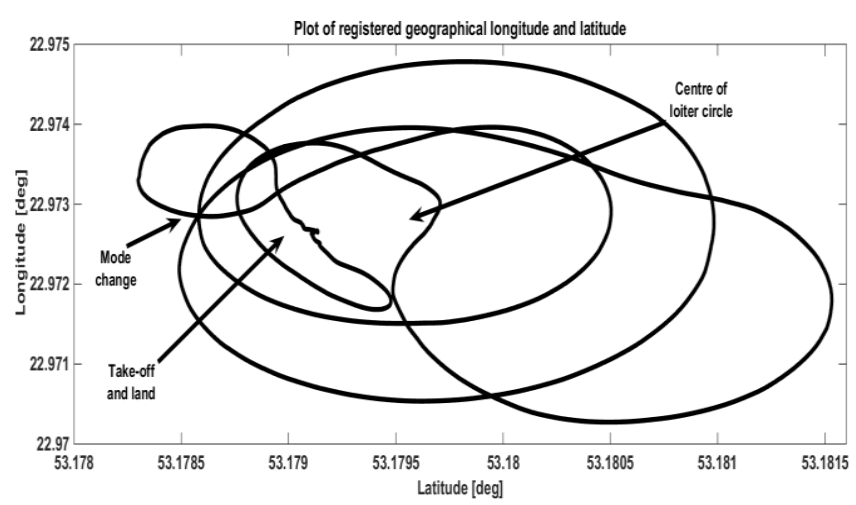

Fig. 9. Plot of registered geographical longitude and latitude for loiter mode of flight. The outer (the biggest) circle was made first, after that wind gusts (around $10[\mathrm{~m} / \mathrm{s}]$ ) affected UAV in way shown above

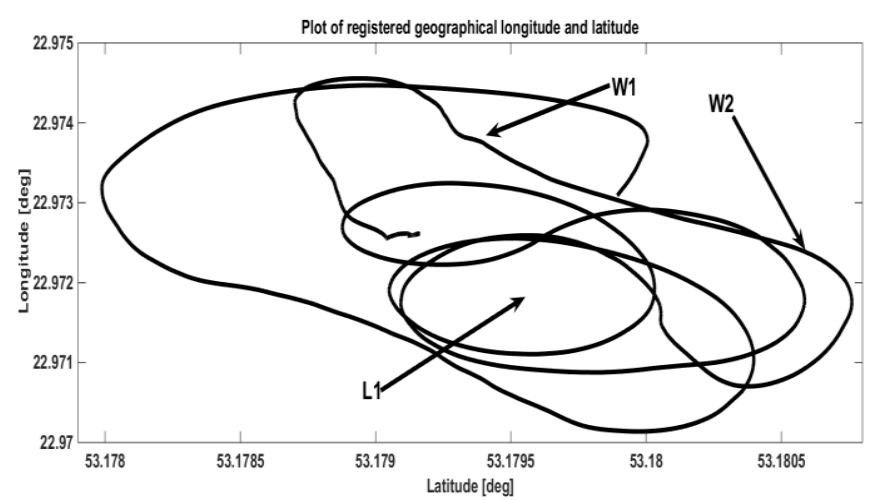

Fig. 10. Plot of registered geographical longitude and latitude for auto and loiter mode. W1 and W2 are waypoints for auto flight mode, L1 mean center of the circle for loiter mode

In the next part of investigations the loiter mode of flight was tested (Fig. 9). It allows for flying in circle of desired radius (in this case $30[\mathrm{~m}]$ ), where the center of circle is determined by geographical longitude and latitude of point where mode setting (by RC controller or GCS application) is done. Also investigations of the auto flight mode in which the autopilot fly between waypoints and altitude $(60[\mathrm{~m}])$ were done (Fig. 10). Take-off and landing were done in manual mode, after reaching safe altitude the mode of flight was changed for ones of described above.

\section{CONCLUSIONS}

The process of preparation and integration of micro airplane platform, flight controller unit and peripheral equipment for realization of different flight modes dedicated for UAV was shown in the paper. Adopted scheme of integration and testing expedite and arrange preparation to UAV to flight. After proper assambly of airplane model with control deflections servos and proppeler drive, the control unit with neccesery equipment was installed on board. Next phase of investigations considered simulation of different modes of flight in software in the loop. During these tests the investigations of chosen flight modes, modification of low-level parameters of flight and ground control station applications were made. Afterwards the field tests of proper autopilot integration and communication systems in flight were made. The more aggressive gain and configuration parameters for roll angle control loop were chosen. In the result more precisely flight on the desired trajectory in loiter mode was achieved. To summarize the integration process of chosen flight control unit on board of micro airplane platform is described in the paper. Used methodology accelerate the whole process and increase safety of field flight tests by performed software in the loop simulations.

\section{REFERENCES}

1. Ambroziak, L., Gosiewski, Z. (2015), Two stage switching control for autonomous formation flight of unmanned aerial vehicles, Aerospace Science and Technology, 46, 221- 226.

2. Crespo G., Glez-de-Rivera G., Garrido J., Ponticelli R.(2014): "Setup of a communication and control systems of a quadrotor type Unmanned Aerial Vehicle", Proceedings of Conference on Design of Circuits and Integrated Circuits (DC/S), Madrid, Spain, 1-6.

3. Erdos D., Erdos A., Watkins S.E. (2013), An experimental UAV system for search and rescue challenge, Aerospace and Electronic Systems Magazine, 28, 32-37.

4. HaiYang C. , YongCan C., YangQuan C. (2010), Autopilots for small unmanned aerial vehicles: A survey, International Journal of Control, Automation and Systems, 8(1), 36-44.

5. http://plane.ardupilot.com/ (access 30.08.2016)

6. http://planner.ardupilot.com/ (access 30.08.2016)

7. Kondratiuk M., Gosiewski Z. (2013), Simulation model of an electromagnetic multi-coil launcher for micro aerial vehicles, Solid State Phenomena: Mechatronic Systems and Materials IV, 406-411.

8. Koslosky E., Wehrmeister M.A., Fabro J.A., Oliveira A.S. (2015), On Using Fuzzy Logic to Control a Simulated Hexacopter Carrying an Attached Pendulum, 2015 Latin America Congress on Computational Intelligence (LA-CCI), 1-6.

9. Koszewnik A. (2014), The Parrot UAV controlled by PID controllers, Acta Mechanica et Automatica, 8(2), 65-69.

10. Kownacki C. (2015), Design of an adaptive Kalman filter to eliminate measurement faults of a laser rangefinder used in the UAV system, Aerospace Science and Technology, 41, 81-89.

11. Kownacki C., Ołdziej D. (2016), Fixed-wing UAVs Flock Control through Cohesion and Repulsion Behaviours Combined with a Leadership, International Journal of Advanced Robotic Systems, 13, 1-10.

12. Mahony R. , Kumar V., Corke P. (2012), Multirotor Aerial Vehicles: Modeling, Estimation, and Control of Quadrotor", IEEE Robotics \& Automation Magazine, 19(3), 20-32.

13. Marconi L., Naldi R., Gentili L. (2011), Modelling and control of a flying robot interacting with the environment, Automatica, 47, 2571-2583. 
15. Meier L., Tanskanen P., Heng L., Lee G. H., Fraundorfer F., Pollefeys M. (2012), PIXHAWK: A micro aerial vehicle design for autonomous flight using onboard computer vision, Autonomous Robots, 33(1-2), 21-39.

16. Multiplex Mentor Assambly Manual http://hitecrcd.com/files/md_mentor_5sp.pdf (access 30.08.2016)

17. Mystkowski A. (2014), Implementation and investigation of a robust control algorithm for an unmanned micro-aerial vehicle, Robotics and Autonomous Systems, 62, 1187-1196.

18. Orifianto O., Farhood M. (2015), Development and Modeling of a Low-Cost Unmanned Aerial Vehicle Research Platform, Journal of Intelligent \& Robotic Systems, 80(1), 139-164.

19. Spinka O., Holub O., Hanzalek Z. (2011) Low-Cost Reconfigurable Control System for Small UAVs, Transactions on Industrial electronics, 58(3), 880-889.
20. Walendziuk W., Sawicki A., Idźkowski A. (2015), Estimation of the object orientation and location with the use of MEMS sensors, SPIE Proceedings, 9662, 1-6.

21. Wang D., Xu J., Yao R. (2006), Simulation system of telemetering and telecontrol for unmanned aerial vehicle, Aerospace and Electronic Systems Magazine, 21, 3-5.

The work has been accomplished under the research project No. MB/WM/15/2016 and S/WM/1/2016 financed by the Ministry of Science and Higher Education 